\title{
Stochastic Delay Population Dynamics under Regime Switching: Global Solutions and Extinction
}

\author{
Zheng Wu, Hao Huang, and Lianglong Wang \\ School of Mathematical Sciences, Anhui University, Hefei 230039, China \\ Correspondence should be addressed to Lianglong Wang; wangll@ahu.edu.cn
}

Received 30 January 2013; Accepted 26 March 2013

Academic Editor: Zhiming Guo

Copyright (C) 2013 Zheng Wu et al. This is an open access article distributed under the Creative Commons Attribution License, which permits unrestricted use, distribution, and reproduction in any medium, provided the original work is properly cited.

This paper is concerned with a delay Lotka-Volterra model under regime switching diffusion in random environment. By using generalized Itô formula, Gronwall inequality and Young's inequality, some sufficient conditions for existence of global positive solutions and stochastically ultimate boundedness are obtained, respectively. Finally, an example is given to illustrate the main results.

\section{Introduction}

The delay differential equation

$$
\frac{d x(t)}{d t}=x(t)(a-b x(t)+c x(t-\tau))
$$

has been used to model the population growth of certain species and is known as the delay Lotka-Volterra model or the delay logistic equation. The delay Lotka-Volterra model for $n$ interacting species is described by the $n$-dimensional delay differential equation

$$
\frac{d x(t)}{d t}=\operatorname{diag}\left(x_{1}(t), \ldots, x_{n}(t)\right)(b+A x(t)+B x(t-\tau)),
$$

where $x=\left(x_{1}, \ldots, x_{n}\right)^{T} \in R^{n}, b=\left(b_{1}, \ldots, b_{n}\right)^{T} \in R_{+}^{n}$, $A=\left(a_{i j}\right)_{n \times n} \in R^{n \times n}$, and $B=\left(b_{i j}\right)_{n \times n} \in R^{n \times n}$. There is an extensive literature concerned with the dynamics of this delay model and have had lots of nice results. We here only mention Ahmad and Rao [1], Bereketoglu and Györi [2], Freedman and Ruan [3], and in particular, the books by Gopalsamy [4], Kolmanovskiĭ and Myshkis [5], and Kuang [6], among many others.

In the equations above, the state $x(t)$ denotes the population sizes of the species. Naturally, we focus on the positive solutions and also require the solutions not to explode at a finite time. To guarantee the positive solutions without explosion (i.e., the global positive solutions), some conditions are in general needed to impose on the system parameters. For example, it is generally assumed that $a>0, b>0$, and $c<b$ for (1) while much more complicated conditions are required on matrices $A$ and $B$ for (2) [7] (and the references cited therein).

On the other hand, population systems are often subject to environmental noise, and the system will change significantly, which may change the dynamics of solutions significantly $[8,9]$. It is therefore necessary to reveal how the noise affects the dynamics of solutions for the delay population systems. In fact, many authors have discussed population systems subject to white noise [7-18]. Recall that the parameter $b_{i}$ in (2) represents the intrinsic growth rate of species $i$. In practice we usually estimate it by an average value plus an error term. According to the well-known central limit theorem, the error term follows a normal distribution. In term of mathematics, we can therefore replace the rate $b_{i}$ by $b_{i}+\sigma_{i} \dot{w}(t)$, where $\dot{w}(t)$ is a white noise (i.e., $w(t)$ is a Brownian motion) and $\sigma_{i} \geq 0$ represents the intensity of noise. As a result, (2) becomes a stochastic differential equation (SDE, in short)

$$
\begin{aligned}
d x(t)= & \operatorname{diag}\left(x_{1}(t), \ldots, x_{n}(t)\right) \\
& \times[(b+A x(t)+B x(t-\tau)) d t+\sigma d w(t)],
\end{aligned}
$$

where $\sigma=\left(\sigma_{1}, \ldots, \sigma_{n}\right)^{T}$. We refer to [7] for more details. 
To our knowledge, much of the attention paid to environmental noise is focused on white noise. But another type of environmental noise, namely, color noise, say telegraph noise, has been studied by many authors ([19-25] and the references cited therein). In this context, telegraph noise can be described as a random switching between two or more environmental regimes, which differ in terms of factors such as nutrition or rain falls $[23,24]$. Usually, the switching between different environments is memoryless and the waiting time for the next switch has an exponential distribution. This indicates that we may model the random environments and other random factors in the system by a continuoustime Markov chain $r(t), t \geq 0$ with a finite state space $S=\{1,2, \ldots, N\}$. Therefore stochastic delay population system (3) in random environments can be described by the following stochastic model with regime switching:

$$
\begin{aligned}
& d x(t)= \operatorname{diag}\left(x_{1}(t), \ldots, x_{n}(t)\right) \\
& \times[(b(r(t))+A(r(t)) x(t)+B(r(t)) x(t-\tau)) d t \\
&\quad+\sigma(r(t)) d w(t)] .
\end{aligned}
$$

The mechanism of ecosystem described by (4) can be explained as follows. Assume that initially, the Markov chain $r(0)=\iota \in S$. Then the ecosystem (4) obeys the SDE

$$
\begin{aligned}
& d x(t) \\
& =\operatorname{diag}\left(x_{1}(t), \ldots, x_{n}(t)\right) \\
& \quad \times[(b(\iota)+A(\iota) x(t)+B(\iota) x(t-\tau)) d t+\sigma(\iota) d w(t)],
\end{aligned}
$$

until the Markov chain $r(t)$ jumps to another state, say, $\varsigma$. Therefore, the ecosystem (4) satisfies the SDE

$$
\begin{aligned}
& d x(t) \\
& =\operatorname{diag}\left(x_{1}(t), \ldots, x_{n}(t)\right) \\
& \quad \times[(b(\varsigma)+A(\varsigma) x(t)+B(\varsigma) x(t-\tau)) d t+\sigma(\varsigma) d w(t)],
\end{aligned}
$$

for a random amount of time until the Markov chain $r(t)$ jumps to a new state again.

It should be pointed out that the stochastic population systems under regime switching have received much attention lately. For instance, the stochastic permanence and extinction of a logistic model under regime switching were considered in $[20,24]$, asymptotic results of a competitive Lotka-Volterra model in random environment are obtain in [25], a new single-species model disturbed by both white noise and colored noise in a polluted environment was developed and analyzed in [26], and a general stochastic logistic system under regime switching was proposed and was treated in [27].

Equation (4) describes the dynamics of populations. This paper is concerned with the positive global solutions, ultimate boundedness and extinction. The stochastic permanence and asymptotic estimations of solutions will be investigated in the next note [28].

This paper is organized as follows. In the next section, some sufficient conditions for global positive solutions for any initial positive value are given by using generalized Itô formula, Gronwall inequality, and $V$-function techniques. In Section 3, the stochastically ultimate boundedness of solutions is obtained by virtue of Young's inequality. Section 4 is devoted to the extinction of solutions. Finally, an example and its numerical simulation are given to illustrate our main results.

\section{Global Positive Solution}

Throughout this paper, unless otherwise specified, let $(\Omega, \mathscr{F}$, $\left.\left\{\mathscr{F}_{t}\right\}_{t \geq 0}, P\right)$ be a complete probability space with a filtration $\left\{\mathscr{F}_{t}\right\}_{t \geq 0}$ satisfying the usual conditions (i.e., it is right continuous and $\mathscr{F}_{0}$ contains all $P$-null sets). Let $w(t), t \geq 0$, be a scalar standard Brownian motion defined on this probability space. We also denote by $R_{+}^{n}$ the positive cone in $R^{n}$, that is $R_{+}^{n}=\left\{x \in R^{n}: x_{i}>0\right.$ for all $\left.1 \leq i \leq n\right\}$, and denote by $\bar{R}_{+}^{n}$ the nonnegative cone in $R^{n}$, that is $\bar{R}_{+}^{n}=\left\{x \in R^{n}: x_{i} \geq\right.$ 0 for all $1 \leq i \leq n\}$. If $A$ is a vector or matrix, its transpose is denoted by $A^{T}$. If $A$ is a matrix, its trace norm is denoted by $|A|=\sqrt{\operatorname{trace}\left(A^{T} A\right)}$, and its operator norm is denoted by $\|A\|=\sup \{|A x|:|x|=1\}$. Moreover, let $\tau>0$ and denote by $C\left([-\tau, 0] ; R_{+}^{n}\right)$ the family of continuous functions from $[-\tau, 0]$ to $R_{+}^{n}$.

In this paper we will use a lot of quadratic functions of the form $x^{T} A x$ for the state $x \in R_{+}^{n}$ only. Therefore, for a symmetric $n \times n$ matrix $A$, we naturally introduce the following definition

$$
\lambda_{\max }^{+}(A)=\sup _{x \in R_{+}^{n},|x|=1} x^{T} A x
$$

For more properties of $\lambda_{\max }^{+}(A)$, refer to the appendix in [7]. Let $r(t)$ be a right-continuous Markov chain on the probability space, taking values in a finite state space $S=$ $\{1,2, \ldots, N\}$, with the generator $\Gamma=\left(\gamma_{u v}\right)$ given by

$$
P\{r(t+\delta)=v \mid r(t)=u\}= \begin{cases}\gamma_{u v} \delta+o(\delta), & \text { if } u \neq v, \\ 1+\gamma_{u v} \delta+o(\delta), & \text { if } u=v\end{cases}
$$

where $\delta>0, \gamma_{u v}$ is the transition rate from $u$ to $v$, and $\gamma_{u v} \geq 0$ if $u \neq v$, while $\gamma_{u u}=-\sum_{v \neq u} \gamma_{u v}$. We assume that the Markov chain $r(\cdot)$ is independent of the Brownian motion $w(\cdot)$. It is well known that almost every sample path of $r(\cdot)$ is a rightcontinuous step function with a finite number of jumps in any finite subinterval of $\bar{R}_{+}$. As a standing hypothesis we assume in this paper that the Markov chain $r(t)$ is irreducible. This is a very reasonable assumption as it means that the system can switch from any regime to any other regime. This is equivalent to the condition that for any $u, v \in S$, one can find finite numbers $i_{1}, i_{2}, \ldots, i_{k} \in S$ such that $\gamma_{u i_{1}} \gamma_{i_{1} i_{2}} \cdots \gamma_{i_{k} v}>0$. Under this condition, the Markov chain has a unique stationary 
(probability) distribution $\pi=\left(\pi_{1}, \pi_{2}, \ldots, \pi_{N}\right) \in R^{1 \times N}$ which can be determined by solving the following linear equation:

$$
\pi \Gamma=0
$$

subject to

$$
\sum_{i=1}^{N} \pi_{i}=1, \quad \pi_{i}>0, \forall i \in S .
$$

We refer to $[12,29]$ for the fundamental theory of stochastic differential equations.

For convenience and simplicity in the following discussion, for any constant sequence $f_{i}(k),(1 \leq i \leq n, k \in S)$ let

$$
\begin{array}{ll}
\check{f}=\max _{1 \leq i \leq n, k \in S} f_{i}(k), & \check{f}(k)=\max _{1 \leq i \leq n} f_{i}(k), \\
\widehat{f}=\min _{1 \leq i \leq n, k \in S} f_{i}(k), & \widehat{f}(k)=\min _{1 \leq i \leq n} f_{i}(k) .
\end{array}
$$

As $x(t)$ in system (4) denotes populations size at time $t$, it should be nonnegative. Thus for further study, we must give some condition under which (4) has a unique global positive solution.

Theorem 1. Assume that there are positive numbers $c_{1}, \ldots, c_{n}$ and $\theta$ such that

$$
\begin{aligned}
\max _{k \in S}\left\{\lambda_{\max }^{+}[\right. & \frac{1}{2} \bar{C}\left(A(k)+A^{T}(k)\right) \bar{C} \\
& \left.\left.+\frac{1}{4 \theta} \bar{C} B(k) B^{T}(k) \bar{C}+\theta I\right]\right\} \leq 0,
\end{aligned}
$$

where $\bar{C}=\operatorname{diag}\left(c_{1}, \ldots, c_{n}\right)$. Then for any given initial data $\{x(t):-\tau \leq t \leq 0\} \in C\left([-\tau, 0] ; R_{+}^{n}\right)$, there is a unique solution $x(t)$ to (4) on $t \geq-\tau$ and the solution will remain in $R_{+}^{n}$ with probability 1 , namely, $x(t) \in R_{+}^{n}$ for all $t \geq-\tau$ a.s.

Proof. Since the coefficients of the equation are locally Lipschitz continuous, for any given initial data $\{x(t):-\tau \leq$ $t \leq 0\} \in C\left([-\tau, 0] ; R_{+}^{n}\right)$, there is a unique maximal local solution $x(t)$ on $t \in\left[-\tau, \tau_{e}\right)$, where $\tau_{e}$ is the explosion time. To show that the solution is global, we need to show that $\tau_{e}=\infty$ a.s.

Let $k_{0}>0$ be sufficiently lager such that

$$
\frac{1}{k_{0}} \leq \min _{-\tau \leq t \leq 0}|x(t)| \leq \max _{-\tau \leq t \leq 0}|x(t)| \leq k_{0} .
$$

For each integer $k \geq k_{0}$, define the stopping time

$$
\begin{array}{r}
\tau_{k}=\inf \left\{t \in\left[0, \tau_{e}\right): x_{i}(t) \notin\left(\frac{1}{k}, k\right)\right. \\
\text { for some } i=1,2, \ldots, n\},
\end{array}
$$

where throughout this paper we set inf $\emptyset=\infty$ (as usual $\emptyset$ denotes the empty set). Clearly, $\tau_{k}$ is increasing as $k \rightarrow \infty$. Set $\tau_{\infty}=\lim _{k \rightarrow \infty} \tau_{k}$, where $\tau_{\infty} \leq \tau_{e}$ a.s. If $\tau_{\infty}=\infty$ a.s., then $\tau_{e}=\infty$ a.s. and $x(t) \in R_{+}^{n}$ a.s. for all $t \geq 0$. In other words, to complete the proof, one should show that $\tau_{\infty}=\infty$ a.s. Define $V: R_{+}^{n} \rightarrow R_{+}$by

$$
V(x)=\sum_{i=1}^{n} c_{i}\left(x_{i}-1-\log x_{i}\right) .
$$

The nonnegativity of this function can be seen from $u-1-$ $\log u \geq 0$ on $u>0$. Let $k \geq k_{0}$ and $T>0$ be arbitrary. For $0 \leq t \leq \tau_{k} \wedge T$, it is easy to see by the generalized Itô formula that

$$
\begin{aligned}
E V\left(x\left(\tau_{k} \wedge t\right)\right)= & V(x(0)) \\
& +E \int_{0}^{\tau_{k} \wedge t} L V(x(s), x(s-\tau), r(s)) d s
\end{aligned}
$$

where $L V: R_{+}^{n} \times R_{+}^{n} \times S \rightarrow R$ is defined by

$$
\begin{aligned}
L V(x, y, k)= & x^{T} \bar{C} b(k)+x^{T} \bar{C} A(k) x+x^{T} \bar{C} B(k) y \\
& -c^{T}(b(k)+A(k) x+B(k) y) \\
& +\frac{1}{2} \sigma^{T}(k) \bar{C} \sigma(k),
\end{aligned}
$$

and $c=\left(c_{1}, \ldots, c_{n}\right)^{T}$. Using condition (12) we compute

$$
\begin{aligned}
x^{T} & \bar{C} A(k) x+x^{T} \bar{C} B(k) y \\
\leq & \frac{1}{2} x^{T}(\bar{C} A(k)+A(k) \bar{C}) x \\
& +\frac{1}{4 \theta} x^{T} \bar{C} B(k) B^{T}(k) \bar{C} x+\theta|y|^{2} \\
= & x^{T}\left[\frac{1}{2}(\bar{C} A(k)+A(k) \bar{C})\right. \\
& \left.\quad+\frac{1}{4 \theta} \bar{C} B(k) B^{T}(k) \bar{C}+\theta I\right] x-\theta|x|^{2}+\theta|y|^{2} \\
\leq & -\theta|x|^{2}+\theta|y|^{2} .
\end{aligned}
$$

Moreover, there is a constant $K_{1}>0$ such that

$$
\begin{aligned}
\max _{k \in S}( & x^{T} \bar{C} b(k)+c^{T} A(k) x+c^{T} B(k) y-c^{T} b(k) \\
& \left.+\frac{1}{2} \sigma^{T}(k) \bar{C} \sigma(k)\right) \\
\leq & K_{1}(1+|x|+|y|) .
\end{aligned}
$$

Substituting these inequalities into (17) yields

$$
L V(x, y, i) \leq K_{1}(1+|x|+|y|)-\theta|x|^{2}+\theta|y|^{2} .
$$

Noticing that $u \leq 2(u-1-\log u)+2$ on $u>0$, we compute

$$
\begin{aligned}
|x| & \leq \sum_{i=1}^{n} x_{i} \leq \sum_{i=1}^{n}\left[2\left(x_{i}-1-\log x_{i}\right)+2\right] \\
& \leq 2 n+\frac{2}{\widehat{c}} \sum_{i=1}^{n} c_{i}\left(x_{i}-1-\log x_{i}\right) \\
& =2 n+\frac{2}{\widehat{c}} V(x) .
\end{aligned}
$$


It follows from (20) and (21) that

$$
L V(x, y, k) \leq K_{2}(1+V(x)+V(y))-\theta|x|^{2}+\theta|y|^{2},
$$

where $K_{2}$ is a positive constant. Substituting this inequality into (16) yields

$$
\begin{aligned}
& E V\left(x\left(\tau_{k} \wedge t\right)\right) \\
& \leq V(x(0))+K_{2} E \int_{0}^{\tau_{k} \wedge t}[1+V(x(s))+V(x(s-\tau))] d s \\
& \quad+E \int_{0}^{\tau_{k} \wedge t}\left[-\theta x^{2}(s)+\theta x^{2}(s-\tau)\right] d s .
\end{aligned}
$$

Compute

$$
\begin{aligned}
& E \int_{0}^{\tau_{k} \wedge t} V(x(s-\tau)) d s \\
& \quad=E \int_{-\tau}^{\tau_{k} \wedge(t-\tau)} V(x(s)) d s \\
& \quad \leq \int_{-\tau}^{0} V(x(s)) d s+E \int_{0}^{\tau_{k} \wedge t} V(x(s)) d s
\end{aligned}
$$

and, similarly

$$
E \int_{0}^{\tau_{k} \wedge t}|x(s-\tau)|^{2} d s \leq \int_{-\tau}^{0}|x(s)|^{2} d s+E \int_{0}^{\tau_{k} \wedge t}|x(s)|^{2} d s .
$$

Substituting these inequalities into (23) gives

$$
\begin{aligned}
E V\left(x\left(\tau_{k} \wedge t\right)\right) & \leq K_{3}+2 K_{2} E \int_{0}^{\tau_{k} \wedge t} V(x(s)) d s \\
& \leq K_{3}+2 K_{2} E \int_{0}^{t} V\left(x\left(\tau_{k} \wedge s\right)\right) d s \\
& \leq K_{3}+2 K_{2} \int_{0}^{t} E V\left(x\left(\tau_{k} \wedge s\right)\right) d s
\end{aligned}
$$

where $K_{3}=V(x(0))+K_{2} T+K_{2} \int_{-\tau}^{0} V(x(s)) d s+$ $\theta \int_{-\tau}^{0}|x(s)|^{2} d s$.

By the Gronwall inequality, we obtain that

$$
E V\left(x\left(\tau_{k} \wedge T\right)\right) \leq K_{3} e^{2 T K_{2}} .
$$

Noting that for every $\omega \in\left\{\tau_{k} \leq T\right\}$,

$$
V\left(x\left(\tau_{k}, \omega\right)\right) \geq \widehat{c}[(k-1-\log k) \wedge(1 / k-1+\log k)],
$$

one has by (27) that

$$
\begin{aligned}
K_{3} e^{2 T K_{2}} \geq & E V\left(x\left(\tau_{k} \wedge T\right)\right) \\
\geq & E\left[1_{\left\{\tau_{k} \leq T\right\}}(\omega) V\left(x\left(\tau_{k} \wedge T, \omega\right)\right)\right] \\
= & E\left[\mathbf{1}_{\left\{\tau_{k} \leq T\right\}}(\omega) V\left(x\left(\tau_{k}, \omega\right)\right)\right] \\
\geq & \widehat{c} P\left\{\tau_{k} \leq T\right\} \\
& \times[(k-1-\log k) \wedge(1 / k-1+\log k)],
\end{aligned}
$$

where $\mathbf{1}_{\left\{\tau_{k} \leq T\right\}}$ is the indicator function of $\left\{\tau_{k} \leq T\right\}$. Letting $k \rightarrow \infty$ gives $\lim _{k \rightarrow \infty} P\left\{\tau_{k} \leq T\right\}=0$ and hence $P\left\{\tau_{\infty} \leq T\right\}=$ 0 . Since $T>0$ is arbitrary, we must have $P\left\{\tau_{\infty}<\infty\right\}=0$, so $P\left\{\tau_{\infty}=\infty\right\}=1$ as required.

Assumption 2. Assume that there exist positive numbers $c_{1}, \ldots, c_{n}$ such that

$$
\max _{k \in S}\left\{\lambda_{\max }^{+}\left[\frac{1}{2}\left(\bar{C} A(k)+A^{T}(k) \bar{C}\right)\right]\right\}+\max _{k \in S}\|\bar{C} B(k)\| \leq 0,
$$

where $\bar{C}=\operatorname{diag}\left(c_{1}, \ldots, c_{n}\right)$.

The following theorem is easy to verify in applications, which will be used in the sections below.

Theorem 3. Under Assumption 2, for any given initial data $\{x(t):-\tau \leq t \leq 0\} \in C\left([-\tau, 0] ; R_{+}^{n}\right)$, there is a unique solution $x(t)$ to (4) on $t \geq-\tau$ and the solution will remain in $R_{+}^{n}$ with probability 1, namely, $x(t) \in R_{+}^{n}$ for all $t \geq-\tau$ a.s.

Proof. Define $V: R_{+}^{n} \rightarrow R_{+}$by $V(x)=\sum_{i=1}^{n} c_{i}\left(x_{i}-1-\log x_{i}\right)$. The non-negativity of this function can be seen from $u-1-$ $\log u \geq 0$ on $u>0$, and then we have (16) and (17).

If $B(k) \neq 0, k \in S$, then $\|\bar{C} B(k)\| \neq 0$. Consequently

$$
\begin{aligned}
x^{T} \bar{C} A(k) x+x^{T} \bar{C} B(k) y \\
\leq \frac{1}{2} x^{T}\left(\bar{C} A(k)+A^{T}(k) \bar{C}\right) x \\
\quad+\frac{1}{2\|\bar{C} B(k)\|} x^{T} \bar{C} B(k) B^{T}(k) \bar{C} x \\
\quad+\frac{1}{2}\|\bar{C} B(k)\||y|^{2} \\
=\frac{1}{2} x^{T}\left(\bar{C} A(k)+A^{T}(k) \bar{C}\right) x \\
\quad+\frac{1}{2}\|\bar{C} B(k)\||x|^{2}+\frac{1}{2}\|\bar{C} B(k)\||y|^{2} .
\end{aligned}
$$

Otherwise $\|\bar{C} B(k)\|=0$ for $B(k)=0, k \in S$. In this case, we also have that

$$
\begin{aligned}
& x^{T} \bar{C} A(k) x+x^{T} \bar{C} B(k) y \\
& \leq \frac{1}{2} x^{T}\left(\bar{C} A(k)+A^{T}(k) \bar{C}\right) x+\frac{1}{2}\|\bar{C} B(k)\||x|^{2} \\
&+\frac{1}{2}\|\bar{C} B(k)\||y|^{2} .
\end{aligned}
$$

Thus,

$$
\begin{aligned}
& x^{T} \bar{C} A(k) x+x^{T} \bar{C} B(k) y \\
& \leq \frac{1}{2} x^{T}(\bar{C} A(k)+A(k) \bar{C}) x+\frac{1}{2}\|\bar{C} B(k)\||x|^{2} \\
&+\frac{1}{2}\|\bar{C} B(k)\||y|^{2} .
\end{aligned}
$$


Denote $\eta=\max _{k \in S}\|\bar{C} B(k)\|$. By (33) and Assumption 2, one has

$$
\begin{aligned}
& x^{T} \bar{C} A(k) x+x^{T} \bar{C} B(k) y \\
& \leq \frac{1}{2} x^{T}(\bar{C} A(k)+A(k) \bar{C}) x+\frac{1}{2} \eta|x|^{2}+\frac{1}{2} \eta|y|^{2} \\
& \leq \max _{k \in S}\left\{\lambda_{\max }^{+}\left[\frac{1}{2}\left(\bar{C} A(k)+A^{T}(k) \bar{C}\right)\right]\right\}|x|^{2} \\
&+\frac{1}{2} \eta|x|^{2}+\frac{1}{2} \eta|y|^{2} \\
& \leq-\frac{1}{2} \eta|x|^{2}+\frac{1}{2} \eta|y|^{2} .
\end{aligned}
$$

The rest of the proof is similar to that of Theorem 1 and omitted.

\section{Ultimate Boundness}

Theorem 3 shows that solutions of the SDE (4) will remain in the positive cone $R_{+}^{n}$. This nice property provides us with a great opportunity to discuss how solutions vary in $R_{+}^{n}$ in detail. In this section, we give the definitions of stochastically ultimate boundedness of the SDE (4) and some sufficient conditions under which solutions of SDE (4) are stochastically ultimate bounded.

Definition 4. The solutions of (4) are called stochastically ultimately bounded, if for any $\varepsilon \in(0,1)$, there exists a positive constant $H=H(\varepsilon)$, such that the solutions of (4) with any positive initial value have the property that

$$
\limsup _{t \rightarrow+\infty} P\{|x(t)|>H\}<\varepsilon .
$$

Assumption 5. Assume that there exist positive numbers $c_{1}, \ldots, c_{n}$ such that

$$
\begin{aligned}
-\lambda= & \max _{k \in S}\left\{\lambda_{\max }^{+}\left[\frac{1}{2}\left(\bar{C} A(k)+A^{T}(k) \bar{C}\right)\right]\right\} \\
& +\max _{k \in S}\|\bar{C} B(k)\|<0,
\end{aligned}
$$

where $\bar{C}=\operatorname{diag}\left(c_{1}, \ldots, c_{n}\right)$.

Theorem 6. Under Assumption 5, for any given initial data $\{x(t):-\tau \leq t \leq 0\} \in C\left([-\tau, 0] ; R_{+}^{n}\right)$ and any given positive constant $p$, there are two positive constants $K_{1}(p)$ and $K_{2}(p)$, such that the solution $x(t)$ of (4) has the properties that

$$
\begin{gathered}
\limsup _{t \rightarrow \infty} E|x(t)|^{p} \leq K_{1}(p), \\
\limsup _{t \rightarrow \infty} \frac{1}{t} \int_{0}^{t} E|x(s)|^{p+1} d s \leq K_{2}(p) .
\end{gathered}
$$

Proof. By Theorem 3, the solution $x(t)$ will remain in $R_{+}^{n}$ for all $t \geq-\tau$ with probability 1 . If $\max _{k \in S}\|\bar{C} B(k)\|>0$, we let $\eta=(p+1)^{-1} \max _{k \in S}\|\bar{C} B(k)\|$ and $\gamma=\tau^{-1} \log ((\lambda+2 \eta) / 2 \eta)>$
0 . Define $V(x, t)=e^{\gamma t}\left(\sum_{i=1}^{n} c_{i} x_{i}\right)^{p}=e^{\gamma t}\left(c^{T} x\right)^{p}$. It has by the generalized Itô formula that

$$
\begin{aligned}
d V(x(t), t)= & L V(x(t), x(t-\tau), t, r(t)) d t \\
& +p e^{\gamma t}\left(c^{T} x(t)\right)^{p-1} x^{T}(t) \bar{C} \sigma(r(t)) d w(t),
\end{aligned}
$$

where $L V: R_{+}^{n} \times R_{+}^{n} \times R_{+} \times S \rightarrow R$ is defined by

$$
\begin{aligned}
& \operatorname{LV}(x, y, t, k) \\
& =e^{\gamma t}\left\{\gamma\left(c^{T} x\right)^{p}+p\left(c^{T} x\right)^{p-1} x^{T} \bar{C}(b(k)+A(k) x+B(k) y)\right. \\
& \left.+\frac{1}{2} p(p-1)\left(c^{T} x\right)^{p-2}\left(x^{T} \bar{C} \sigma(k)\right)^{2}\right\} .
\end{aligned}
$$

Meanwhile, by Assumption 5 and Young's inequality, one gets

$$
\begin{aligned}
& L V(x, y, t, k) \\
& \leq e^{\gamma t}\left[\gamma|c|^{p}|x|^{p}+p|c|^{p}|b(k)||x|^{p}\right. \\
& +\frac{1}{2} p(p-1)|c|^{p}|\sigma(k)|^{2}|x|^{p} \\
& \left.+p\left(c^{T} x\right)^{p-1} x^{T} \bar{C}(A(k) x+B(k) y)\right] \\
& \leq e^{\gamma t}\left\{K(p)|x|^{p}+\frac{1}{2} p\left(c^{T} x\right)^{p-1} x\right. \\
& \times\left(\bar{C} A(k)+A^{T}(k) \bar{C}\right) x^{T} \\
& \left.+p\left(c^{T} x\right)^{p-1}\|\bar{C} B(k)\||x||y|\right\} \\
& \leq e^{\gamma t} K(p)|x|^{p}+e^{\gamma t} p|c|^{p-1} \\
& \times \max _{k \in S}\left\{\lambda_{\max }^{+}\left[\frac{1}{2}\left(\bar{C} A(k)+A^{T}(k) \bar{C}\right)\right]\right\}|x|^{p+1} \\
& +e^{\gamma t} p|c|^{p-1}\|\bar{C} B(k)\||x|^{p}|y| \\
& \leq e^{\gamma t} K(p)|x|^{p}+e^{\gamma t} p|c|^{p-1} \\
& \times \max _{k \in S}\left\{\lambda_{\max }^{+}\left[\frac{1}{2}\left(\bar{C} A(k)+A^{T}(k) \bar{C}\right)\right]\right\}|x|^{p+1} \\
& +e^{\gamma t} p|c|^{p-1}\|\bar{C} B(k)\|\left(\frac{p}{p+1}|x|^{p+1}+\frac{1}{p+1}|y|^{p+1}\right) \\
& \leq e^{\gamma t}\left\{K(p)|x|^{p}+p|c|^{p-1}\left[-(\lambda+\eta)|x|^{p+1}+\eta|y|^{p+1}\right]\right\} \\
& \leq e^{\gamma t}\left\{K(p)|x|^{p}-\frac{1}{2} p \lambda|c|^{p-1}|x|^{p+1}+p|c|^{p-1}\right. \\
& \left.\times\left[-\left(\frac{1}{2} \lambda+\eta\right)|x|^{p+1}+\eta|y|^{p+1}\right]\right\} \\
& \leq H(p) e^{\gamma t}+p \eta|c|^{p-1} e^{\gamma t}\left(-e^{\gamma \tau}|x|^{p+1}+|y|^{p+1}\right),
\end{aligned}
$$


where

$$
\begin{gathered}
K(p)=\max _{k \in S}\left[\gamma|c|^{p}+p|c|^{p}|b(k)|+\frac{1}{2} p(p-1)|c|^{p}|\sigma(k)|^{2}\right], \\
H(p)=\sup _{x \in R_{+}}\left(K(p)|x|^{p}-\frac{1}{2} p \lambda|c|^{p-1}|x|^{p+1}\right) \vee 1 .
\end{gathered}
$$

On the other hand,

$$
\begin{aligned}
& \int_{0}^{t} e^{\gamma s}|x(s-\tau)|^{p+1} d s \\
& \quad=e^{\gamma \tau} \int_{0}^{t} e^{\gamma(s-\tau)}|x(s-\tau)|^{p+1} d s \\
& \quad=e^{\gamma \tau} \int_{-\tau}^{t-\tau} e^{\gamma s}|x(s)|^{p+1} d s \\
& \leq e^{\gamma \tau} \int_{-\tau}^{0}|x(s)|^{p+1} d s+e^{\gamma \tau} \int_{0}^{t} e^{\gamma s}|x(s)|^{p+1} d s,
\end{aligned}
$$

by (41) and (43), we obtain that

$$
\begin{aligned}
& e^{\gamma t} E[V(x(t))] \\
& \leq V(x(0))+\int_{0}^{t} H(p) e^{\gamma s} d s+p|c|^{p-1} \eta \\
& \quad \times \int_{0}^{t} e^{\gamma s}\left(-e^{\gamma \tau}|x(s)|^{p+1}+|x(s-\tau)|^{p+1}\right) d s \\
& \leq V(x(0))+\frac{H(p)}{\gamma}\left(e^{\gamma t}-1\right)+p|c|^{p-1} \eta e^{\gamma \tau} \int_{-\tau}^{0}|x(s)|^{p+1} d s,
\end{aligned}
$$

which yields

$$
\limsup _{t \rightarrow \infty} E V(x(t)) \leq \frac{H(p)}{\gamma} .
$$

Since $|x(t)| \leq \sum_{i=1}^{n} x_{i}(t) \leq V(x(t)) / \widehat{c}$, it has $\limsup _{t \rightarrow \infty}$ $E|x(t)|^{p} \leq H(p) / \widehat{c} \gamma$ and the desired assertion (37) follows by setting $K_{1}(p)=H(p) / \widehat{c} \gamma$. It is easy to verify this result, if $\max _{k \in S}\|\bar{C} B(k)\|=0$. We omit its proof here.

Define $\bar{V}(x)=\left(c^{T} x\right)^{p}$. By the generalized Itô formula, it follows

$$
\begin{aligned}
d \bar{V}(x(t))= & L \bar{V}(x(t), x(t-\tau), r(t)) d t \\
& +p\left(c^{T} x(t)\right)^{p-1} x^{T}(t) \bar{C} \sigma(r(t)) d w(t)
\end{aligned}
$$

where $L \bar{V}: R_{+}^{n} \times R_{+}^{n} \times S \rightarrow R$ is defined by

$$
\begin{aligned}
L \bar{V}(x, y, k)= & p\left(c^{T} x\right)^{p-1} x^{T} \bar{C}[b(k)+A(k) x+B(k) y] \\
& +\frac{1}{2} p(p-1)\left(c^{T} x\right)^{p-2}\left(x^{T} \bar{C} \sigma(k)\right)^{2} .
\end{aligned}
$$

By Assumption 5 and Young's inequality again,

$$
\begin{aligned}
& L \bar{V}(x, y, k) \\
& \leq p|c|^{p}|b(k)||x|^{p}+\frac{1}{2} p(p-1)|c|^{p}|\sigma(k)|^{2}|x|^{p} \\
& \quad+p\left(c^{T} x\right)^{p-1} x^{T} \bar{C}(A(k) x+B(k)) y \\
& \leq p|c|^{p}|b(k)||x|^{p}+\frac{1}{2} p(p-1)|c|^{p}|\sigma(k)|^{2}|x|^{p} \\
& \quad+p|c|^{p-1}\left[-(\lambda+\eta)|x|^{p+1}+\eta|y|^{p+1}\right] .
\end{aligned}
$$

It is easy to compute

$$
\begin{aligned}
0 \leq E \bar{V}(x(0)) & \\
+E \int_{0}^{t}[ & {\left[\check{b}|c|^{p}|x(s)|^{p}\right.} \\
& +\frac{1}{2} p|p-1||c|^{p} \check{\sigma}^{2}|x(s)|^{p} \\
& -p|c|^{p-1}(\lambda+\eta)|x(s)|^{p+1} \\
& \left.+p|c|^{p-1} \eta|x(s-\tau)|^{p+1}\right] d s .
\end{aligned}
$$

Moreover,

$$
\int_{0}^{t}|x(s-\tau)|^{p+1} d s \leq \int_{-\tau}^{0}|x(s)|^{p+1} d s+\int_{0}^{t}|x(s)|^{p+1} d s,
$$

hence, we get

$$
\begin{aligned}
& \left.\frac{1}{2} \lambda p|c|\right|^{p-1} E \int_{0}^{t}|x(s)|^{p+1} d s \\
& \leq E \bar{V}(x(0)) \\
& +E \int_{0}^{t}\left[p|c|^{p} \check{b}|x(s)|^{p}\right. \\
& +\frac{1}{2} p|p-1||c|^{p} \check{\sigma}^{2}|x(s)|^{p} \\
& -p\left(\frac{\lambda}{2}+\eta\right)|c|^{p-1}|x(s)|^{p+1} \\
& \left.+p \eta|c|^{p-1}|x(s-\tau)|^{p+1}\right] d s \\
& \leq E \bar{V}(x(0))+p \eta|c|^{p-1} \int_{-\tau}^{0}|x(s)|^{p+1} d s \\
& +E \int_{0}^{t}\left(p|c|^{p} \check{b}|x(s)|^{p}+\frac{1}{2} p|p-1||c|^{p} \check{\sigma}^{2}|x(s)|^{p}\right. \\
& \left.-\frac{1}{2} p \lambda|c|^{p-1}|x(s)|^{p+1}\right) d s \\
& \leq E \bar{V}(x(0))+p \eta|c|^{p-1} \\
& \times \int_{-\tau}^{0}|x(s)|^{p+1} d s+H(p) t,
\end{aligned}
$$


where $H(p)=\sup _{x \in R_{+}}\left(p|c|^{p} \check{b}|x|^{p}+(1 / 2) p|p-1||c|^{p} \check{\sigma}^{2}\right.$ $\left.|x(s)|^{p}-(1 / 2) \lambda p|c|^{p-1}|x|^{p+1}\right)$. This implies immediately that

$$
\limsup _{t \rightarrow \infty} \frac{1}{t} E \int_{0}^{t}|x(s)|^{p+1} d s \leq \frac{2 H(p)}{p \lambda|c|^{p-1}}
$$

and the desired assertion (38) follows by setting $K_{2}(p)=$ $2 H(p) / p \lambda|c|^{p-1}$.

Remark 7. From (37) of Theorem 6, there is a $T>0$ such that

$$
E|x(t)|^{p} \leq 2 K_{1}(p), \quad \forall t \geq T .
$$

Since $E|x(t)|^{p}$ is continuous, there is a $\bar{K}_{1}\left(p, x_{0}\right)$ such that

$$
E|x(t)|^{p} \leq \bar{K}_{1}\left(p, x_{0}\right) \quad \text { for } t \in[0, T] .
$$

Let $L\left(p, x_{0}\right)=\max \left(2 K_{1}(p), \bar{K}_{1}\left(p, x_{0}\right)\right)$, we have

$$
E|x(t)|^{p} \leq L\left(p, x_{0}\right), \quad \forall t \in[0, \infty),
$$

which implies that the pth moment of any positive solution of (4) is bounded.

Remark 8. Conclusion (38) of Theorem 6 shows that the average in time of the $p$ th $(p>1)$ moment of solutions of (4) will be bounded.

Theorem 9. Solutions of (4) are stochastically ultimately bounded under Assumption 5.

Proof. This can be easily verified by Chebyshev's inequality and Theorem 6.

\section{Extinction}

Assumption 10. Assume that there exist positive numbers $c_{1}, \ldots, c_{n}$ such that

$$
\begin{aligned}
& |c|^{-1} \max _{k \in S}\left\{\lambda_{\max }^{+}\left[\frac{1}{2}(\bar{C} A(k)+A(k) \bar{C})\right]\right\} \\
& +\widehat{c}^{-1} \max _{k \in S}\|\bar{C} B(k)\| \leq 0,
\end{aligned}
$$

where $\bar{C}=\operatorname{diag}\left(c_{1}, \ldots, c_{n}\right)$ and $\widehat{c}=\min _{1 \leq i \leq n} c_{i}$.

Theorem 11. Under Assumption 10, for any given initial data $\{x(t):-\tau \leq t \leq 0\} \in C\left([-\tau, 0] ; R_{+}^{n}\right)$, the solution $x(t)$ of $(4)$ has the properties that

$$
\limsup _{t \rightarrow \infty} \frac{1}{t} \log |x(t)| \leq \sum_{i=1}^{n} \pi_{k} \beta(k) \quad \text { a.s., }
$$

where $\beta(k)=\check{b}(k)-(1 / 2) \widehat{\sigma}^{2}(k)$. Particularly, if $\sum_{k=1}^{N} \pi_{k} \beta(k)<$ 0 , then

$$
\limsup _{t \rightarrow \infty} \frac{1}{t} \log |x(t)|<0 \quad \text { a.s. }
$$

That is, the population will become extinct exponentially with probability 1 .
Proof. By Theorem 3, the solution $x(t)$ will remain in $R_{+}^{n}$ for all $t \geq-\tau$ with probability 1 . Define

$$
V(x)=c^{T} x=\sum_{i=1}^{n} c_{i} x_{i} \quad \text { on } x \in R_{+}^{n},
$$

where $c=\left(c_{1}, \ldots, c_{n}\right)^{T}$. Then

$$
\begin{gathered}
d V(x(t))=x^{T}(t) \bar{C}[(b(r(t)+A(r(t)) x(t) \\
+B(r(t)) x(t-\tau))) d t \\
+\sigma(r(t)) d w(t)] .
\end{gathered}
$$

By the generalized Itô formula,

$$
\begin{aligned}
& d \log V(x(t)) \\
& =\frac{1}{V(x(t))} d V(x(t))-\frac{1}{2 V^{2}(x(t))}(d V(x(t)))^{2} \\
& =\frac{1}{V(x(t))} x^{T}(t) \bar{C} \\
& \quad \times[(b(r(t)+A(r(t)) x(t)+B(r(t)) x(t-\tau))) d t \\
& \quad+\sigma(r(t)) d w(t)] \\
& \quad-\frac{1}{2 V^{2}(x(t))}\left|x^{T}(t) \bar{C} \sigma(r(t))\right|^{2} d t .
\end{aligned}
$$

It is computed

$$
\begin{aligned}
& \frac{x^{T}(t) \bar{C} A(r(t)) x(t)}{V(x(t))}+\frac{x^{T}(t) \bar{C} B(r(t)) x(t-\tau)}{V(x(t))} \\
& \leq \frac{x^{T}(t)\left(\bar{C} A(r(t))+A^{T}(r(t)) \bar{C}\right) x(t)}{2 V(x(t))} \\
& +\frac{\|\bar{C} B(r(t))\||x(t-\tau)|}{\widehat{c}} \\
& \leq\left(|c|^{-1} \max _{k \in S}\left\{\lambda_{\max }^{+}\left[\frac{1}{2}(\bar{C} A(k)+A(k) \bar{C})\right]\right\}\right. \\
& \left.\quad+\widehat{c}^{-1} \max _{k \in S}(\|\bar{C} B(k)\|)\right)|x(t)| \\
& +\widehat{c}^{-1} \max _{k \in S}\|\bar{C} B(k)\|(-|x(t)|+|x(t-\tau)|), \\
& \frac{x^{T}(t) \bar{C} b(r(t))}{V(x(t))}-\frac{\left|x^{T}(t) \bar{C} \sigma(r(t))\right|^{2}}{2 V^{2}(t)} \\
& \quad \leq \check{b}(r(t))-\frac{1}{2} \widehat{\sigma}^{2}(r(t))=\beta(r(t)) .
\end{aligned}
$$


Substituting these two inequalities into (61) yields

$$
\begin{aligned}
& \log V(x(t)) \\
& \leq \log V(x(0))+\int_{0}^{t} \beta(r(s)) d s+\widehat{c}^{-1} \max _{k \in S}\|\bar{C} B(k)\| \\
& \quad \times \int_{0}^{t}[-|x(s)|+|x(s-\tau)|] d s+M(t) \\
& \leq \log V(x(0))+\widehat{c}^{-1} \max _{k \in S}\|\bar{C} B(k)\| \int_{-\tau}^{0} x(s) d s \\
& \quad+\int_{0}^{t} \beta(r(s)) d s+M(t),
\end{aligned}
$$

where $M(t)$ is a martingale defined by

$$
M(t)=\int_{0}^{t} \frac{x^{T}(s) \bar{C} \sigma(r(s))}{V(x(s))} d w(t) .
$$

The quadratic variation of this martingale is

$$
\langle M, M\rangle_{t}=\int_{0}^{t} \frac{\left|x^{T}(s) \bar{C} \sigma(r(s))\right|^{2}}{V^{2}(x(s))} d s \leq \check{\sigma}^{2} t,
$$

hence

$$
\limsup _{t \rightarrow \infty} \frac{\langle M, M\rangle_{t}}{t} \leq \check{\sigma}^{2} \quad \text { a.s. }
$$

Applying the strong law of large numbers for martingales [29], we therefore have

$$
\lim _{t \rightarrow \infty} \frac{M(t)}{t}=0 \quad \text { a.s. }
$$

It finally follows from (64) by dividing $t$ on the both sides and then letting $t \rightarrow \infty$ that

$$
\begin{aligned}
\limsup _{t \rightarrow \infty} \frac{\log V(x(t))}{t} & \leq \underset{t \rightarrow \infty}{\limsup } \frac{1}{t} \int_{0}^{t} \beta(r(s)) d s \\
& =\sum_{k=1}^{N} \pi_{k} \beta(k) \quad \text { a.s., }
\end{aligned}
$$

which is the required assertion (57).

Similarly, we can prove the following conclusions.

Theorem 12. Assume that Assumption 10 holds. Assume moreover that the noise intensities $\sigma(i)$ are sufficiently large in the sense that

$$
\begin{gathered}
\sigma_{i}(k) \sigma_{j}(k)-b_{i}(k)-b_{j}(k)>0, \\
1 \leq i, j \leq n, \text { for each } k \in S,
\end{gathered}
$$

then for any given initial data $\{x(t):-\tau \leq t \leq 0\} \in$ $C\left([-\tau, 0] ; R_{+}^{n}\right)$, the solution $x(t)$ of $(4)$ has the properties that

$$
\limsup _{t \rightarrow \infty} \frac{1}{t} \log |x(t)| \leq-\frac{1}{2} \sum_{k=1}^{N} \pi_{k} \varphi(k) \quad \text { a.s., }
$$

where

$$
\varphi(k)=\min _{1 \leq i, j \leq n}\left(\sigma_{i}(k) \sigma_{j}(k)-b_{i}(k)-b_{j}(k)\right)>0 .
$$

That is, the population will become extinct exponentially with probability 1 .

Proof. Let $V: R_{+}^{n} \rightarrow R_{+}$be the same as defined in the proof of Theorem 11, so we have (60), (61), and (62). It is also computed

$$
\begin{aligned}
& \frac{x^{T}(t) \bar{C} b(r(t))}{V(x(t))}-\frac{\left|x^{T}(x(t)) \bar{C} \sigma(r(t))\right|^{2}}{2 V^{2}(x(t))} \\
= & \frac{2 x^{T}(t) \bar{C} b(r(t)) c^{T} x(t)}{2 V^{2}(x(t))} \\
& -\frac{x^{T}(t) \bar{C} \sigma(r(t)) \sigma^{T}(r(t)) \bar{C} x(t)}{2 V^{2}(x(t))} \\
= & \frac{2 x^{T}(t) \bar{C} b(r(t)) \overrightarrow{1} \bar{C} x(t)}{2 V^{2}(x(t))} \\
& -\frac{x^{T}(t) \bar{C} \sigma(r(t)) \sigma^{T}(r(t)) \bar{C} x(t)}{2 V^{2}(x(t))} \\
= & \frac{x^{T}(t) \bar{C} b(r(t)) \overrightarrow{1}+\overrightarrow{1}^{T} b^{T}(r(t)) \bar{C} x(t)}{2 V^{2}(x(t))} \\
& -\frac{x^{T}(t) \bar{C} \sigma(r(t)) \sigma^{T}(r(t)) \bar{C} x(t)}{2 V^{2}(x(t))} \\
= & -\frac{x^{T}(t) \bar{C} Q(r(t)) \bar{C} x(t)}{2 V^{2}(x(t))},
\end{aligned}
$$

where $\overrightarrow{1}=(1, \ldots, 1)$ and $Q(k)=\sigma(k) \sigma^{T}(k)-(b(k) \overrightarrow{1}+$ $\left.\overrightarrow{1}^{T} b^{T}(k)\right)$. Substituting (62) and (73) into (61) yields

$$
\begin{aligned}
& \log V(x(t)) \\
& \leq \log V(x(0))-\int_{0}^{t} \frac{x^{T}(s) \bar{C} Q(r(s)) \bar{C} x(s)}{2 V^{2}(x(s))} d s \\
& \quad+\widehat{c}^{-1} \max _{k \in S}\|\bar{C} B(k)\| \int_{0}^{t}[-|x(s)|+|x(s-\tau)|] d s+M(t) .
\end{aligned}
$$

Note that $\sigma_{i}(k) \sigma_{j}(k)-b_{i}(k)-b_{j}(k)$, the $i j$ th element of the matrix $Q(k)$ is positive by (70). It is therefore easy to verify

$$
x^{T}(t) \bar{C} Q(k) \bar{C} x(t) \geq \varphi(k) V^{2}(x(t)),
$$

where $\varphi(\cdot)$ has been defined in the statement of the theorem. Substituting this inequality into (74) yields

$$
\begin{aligned}
& \log V(x(t)) \\
& \leq \log V(x(0))-\int_{0}^{t} \frac{1}{2} \varphi(k) d s+\widehat{c}^{-1} \max _{k \in S}\|\bar{C} B(k)\| \\
& \quad \times \int_{0}^{t}(-|x(s)|+|x(s-\tau)|) d s+M(t) .
\end{aligned}
$$


The rest of the proof is similar to that of Theorem 11 and omitted.

\section{Examples}

In this section, an example and corresponding numerical simulations are given to illustrate our main results.

Example 13. Consider the two-species Lotka-Volterra system with regime switching described by

$$
\begin{aligned}
d x(t)= & \operatorname{diag}\left(x_{1}(t), x_{2}(t)\right) \\
& \times[(b(r(t))+A(r(t)) x(t) \\
& \quad+B(r(t)) x(t-\tau)) d t+\sigma(r(t)) d w(t)],
\end{aligned}
$$

where $x(t)=\left(x_{1}(t), x_{2}(t)\right)^{T}, b(r(t))=\left(b_{1}(r(t)), b_{2}(r(t))\right)^{T}$, $\sigma(r(t))=\left(\sigma_{1}(r(t)), \sigma_{2}(r(t))\right)^{T}$,

$$
\begin{gathered}
A(r(t))=\left(\begin{array}{ll}
a_{11}(r(t)) & a_{12}(r(t)) \\
a_{21}(r(t)) & a_{22}(r(t))
\end{array}\right), \\
B(r(t))=\left(\begin{array}{ll}
b_{11}(r(t)) & b_{12}(r(t)) \\
b_{21}(r(t)) & b_{22}(r(t))
\end{array}\right)
\end{gathered}
$$

and $r(t)$ is a right-contiuous Markov chain taking values in $S=\{1,2\}$, and $r(t)$ and $w(t)$ are independent. Here

$$
\begin{array}{lll}
b_{1}(1)=5, & a_{11}(1)=-5, & a_{12}(1)=3, \\
b_{11}(1)=0, & b_{12}(1)=\frac{1}{2}, & \sigma_{1}(1)=\sqrt{2}, \\
b_{2}(1)=8, & a_{21}(1)=3, & a_{22}(1)=-5, \\
b_{21}(1)=1, & b_{22}(1)=0, & \sigma_{2}(1)=2, \\
b_{1}(2)=4, & a_{11}(2)=-3, & a_{12}(2)=1, \\
b_{11}(2)=0, & b_{12}(2)=1, & \sigma_{1}(2)=\sqrt{14}, \\
b_{2}(2)=5, & a_{21}(2)=1, & a_{22}(2)=-3, \\
b_{21}(2)=\frac{1}{2}, & b_{22}(2)=0, & \sigma_{2}(2)=4 .
\end{array}
$$

Let $\bar{C}=I \in R^{2 \times 2}$, the identity matrix. It is easy to compute

$$
\begin{gathered}
|c|=\sqrt{2}, \quad \widehat{c}=1, \quad \beta(1)=7, \quad \beta(2)=-2, \\
\max _{k \in S} \lambda_{\max }^{+}\left[\frac{1}{2}\left(\bar{C} A(k)+A^{T}(k) \bar{C}\right)\right] \leq-2, \\
\max _{k \in S}\|\bar{C} B(k)\| \leq \frac{\sqrt{5}}{2} .
\end{gathered}
$$

Then

$$
\begin{aligned}
|c|^{-1} \max _{k \in S}\left\{\lambda_{\max }^{+}\left[\frac{1}{2}\left(\bar{C} A(k)+A^{T}(k) \bar{C}\right)\right]\right\} \\
+\widehat{c}^{-1} \max _{k \in S}\|\bar{C} B(k)\|<0 .
\end{aligned}
$$

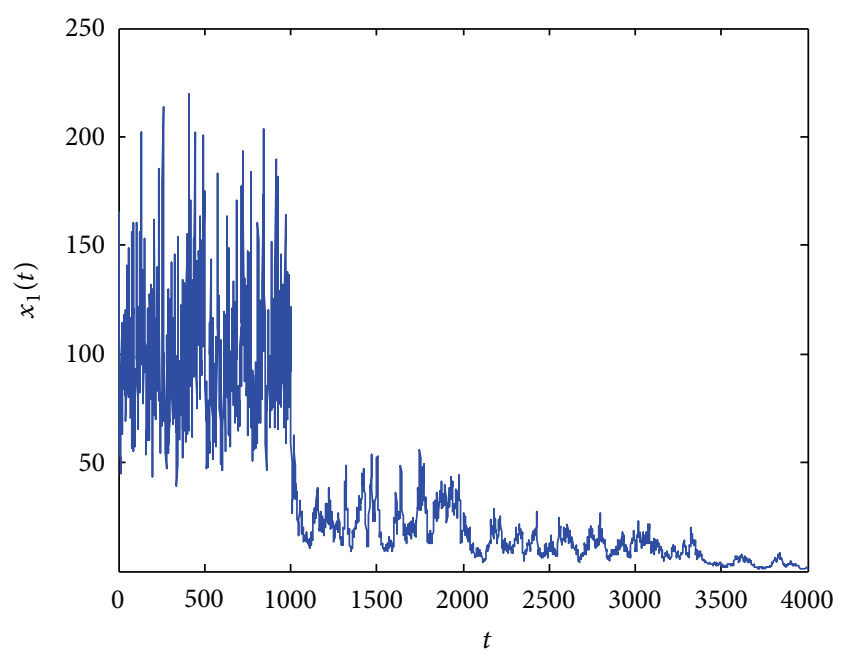

(a)

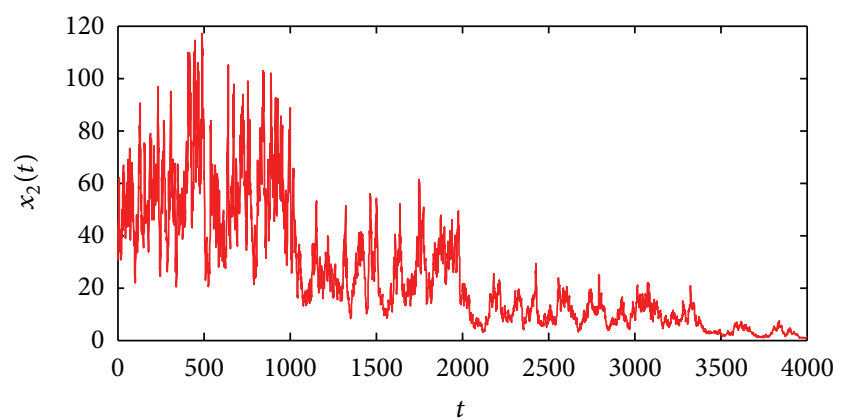

(b)

Figure 1

By Theorems 3 and 9, the solutions of (77) will remain in $R_{+}^{2}$ for all $t \geq-\tau$ with probability 1 and are stochastically ultimately bounded.

Let the generator of the Markov chain $r(t)$ be

$$
\Gamma=\left(\begin{array}{rr}
-4 & 4 \\
1 & -1
\end{array}\right)
$$

By solving the linear equation $\pi \Gamma=0$, we obtain the unique stationary (probability) distribution $\pi=\left(\pi_{1}, \pi_{2}\right)=$ $(1 / 5,4 / 5)$. Then $\sum_{k=1}^{2} \pi_{k} \beta(k)=-1 / 5<0$. Therefore, by Theorems 11, (77) is extinctive, shown in Figure 1.

In Figure 1, for numerical solutions of (77), step size $\Delta t=$ 0.001 , delay $\tau=1$. Initial datum of $\left(x_{1}(t), x_{2}(t)\right)$ are random numbers in $[1,200] \times[1,600]$. Initial datum are not shown in Figure 1.

\section{Conclusion}

This work is concerned with delay Lotka-Volterra model under regime switching diffusion in random environment. It should be pointed out that (77) is more difficult to handle than (3) in [23]. Fortunately, the difficulties caused by delay term are overcome by using Young's inequality. The model in [7] is similar to (4), while the coefficients in (4) are varied with 
regime switching. Similar results are technically obtained by making use of comparison principle.

\section{Acknowledgments}

The authors are grateful to the editor Prof. Zhiming Guo and anonymous referees for their helpful comments and suggestions which have improved the quality of this paper. The authors are indebted to Professor Tao Wu, Anhui University, for giving ideas on simulations of the example. This work is supported by Research Fund for Doctor Station of Ministry of Education of China (no. 20113401110001, no. 20103401120002), TIAN YUAN Series of Natural Science Foundation of China (no. 11126177, No. 11226247), Key Natural Science Foundation (no. KJ2009A49, no. KJ20 12A19), 211 Project of Anhui University (no. KJJQ1101), Anhui Provincial Nature Science Foundation (no. 1308085MA01, no. 1308085QA15, and no. 1208085QA15), Foundation for Young Talents in College of Anhui Province (no. 2012SQRL021).

\section{References}

[1] S. Ahmad and M. R. M. Rao, "Asymptotically periodic solutions of $N$-competing species problem with time delays," Journal of Mathematical Analysis and Applications, vol. 186, no. 2, pp. 559$571,1994$.

[2] H. Bereketoglu and I. Győri, "Global asymptotic stability in a nonautonomous Lotka-Volterra type system with infinite delay," Journal of Mathematical Analysis and Applications, vol. 210, no. 1, pp. 279-291, 1997.

[3] H. I. Freedman and S. G. Ruan, "Uniform persistence in functional-differential equations," Journal of Differential Equations, vol. 115, no. 1, pp. 173-192, 1995.

[4] K. Gopalsamy, Stability and Oscillations in Delay Differential Equations of Population Dynamics, vol. 74 of Mathematics and its Applications, Kluwer Academic, Dordrecht, The Netherlands, 1992.

[5] V. Kolmanovskii and A. Myshkis, Applied Theory of FunctionalDifferential Equations, vol. 85, Kluwer Academic, Dordrecht, The Netherlands, 1992.

[6] Y. Kuang, Delay Differential Equations with Applications in Population Dynamics, vol. 191 of Mathematics in Science and Engineering, Academic Press, Boston, MA, USA, 1993.

[7] A. Bahar and X. Mao, "Stochastic delay population dynamics," International Journal of Pure and Applied Mathematics, vol. 11, no. 4, pp. 377-400, 2004.

[8] X. Mao, S. Sabanis, and E. Renshaw, "Asymptotic behaviour of the stochastic Lotka-Volterra model," Journal of Mathematical Analysis and Applications, vol. 287, no. 1, pp. 141-156, 2003.

[9] X. Mao, G. Marion, and E. Renshaw, "Environmental Brownian noise suppresses explosions in population dynamics," Stochastic Processes and their Applications, vol. 97, no. 1, pp. 95-110, 2002.

[10] D. Jiang and N. Shi, "A note on nonautonomous logistic equation with random perturbation," Journal of Mathematical Analysis and Applications, vol. 303, no. 1, pp. 164-172, 2005.

[11] D. Jiang, N. Shi, and X. Li, "Global stability and stochastic permanence of a non-autonomous logistic equation with random perturbation," Journal of Mathematical Analysis and Applications, vol. 340, no. 1, pp. 588-597, 2008.
[12] T. C. Gard, Introduction to stochastic differential equations, vol. 114 of Monographs and Textbooks in Pure and Applied Mathematics, Marcel Dekker, New York, NY, USA, 1988.

[13] A. Bahar and X. Mao, "Stochastic delay Lotka-Volterra model," Journal of Mathematical Analysis and Applications, vol. 292, no. 2, pp. 364-380, 2004.

[14] X. Mao, "Delay population dynamics and environmental noise," Stochastics and Dynamics, vol. 5, no. 2, pp. 149-162, 2005.

[15] S. Pang, F. Deng, and X. Mao, "Asymptotic properties of stochastic population dynamics," Dynamics of Continuous, Discrete \& Impulsive Systems A, vol. 15, no. 5, pp. 603-620, 2008.

[16] H. Huang, Z. Wu, and L. Wang, " $\psi^{\gamma}$ stability analysis for neutral stochastic neural networks withmultiple delays based on LMI approach" (Chinese), Journal of Biomathmatics. In press.

[17] Z. Wu, H. Huang, and L. Wang, "Dynamical behavior of a stochastic ratio-dependent predator-prey system," Journal of Applied Mathematics, Article ID 857134, 17 pages, 2012.

[18] Z. Wu, H. Huang, and L. Wang, "Exponential stability of impulsive stochastic functional differential systems," Abstract and Applied Analysis, vol. 2012, Article ID 678536, 12 pages, 2012.

[19] Z. Wu, H. Huang, and L. Wang, "Stochastic delay logistic model with Markovian switching," International Journal of Information and Systems Science, vol. 8, no. 2, pp. 174-180, 2012.

[20] Z. Wu, H. Huang, and L. Wang, "Stochastic delay logistic model under regime switching," Abstract and Applied Analysis, vol. 2012, Article ID 241702, 26 pages, 2012.

[21] Y. Takeuchi, N. H. Du, N. T. Hieu, and K. Sato, "Evolution of predator-prey systems described by a Lotka-Volterra equation under random environment," Journal of Mathematical Analysis and Applications, vol. 323, no. 2, pp. 938-957, 2006.

[22] Q. Luo and X. Mao, "Stochastic population dynamics under regime switching," Journal of Mathematical Analysis and Applications, vol. 334, no. 1, pp. 69-84, 2007.

[23] X. Li, D. Jiang, and X. Mao, "Population dynamical behavior of Lotka-Volterra system under regime switching," Journal of Computational and Applied Mathematics, vol. 232, no. 2, pp. 427-448, 2009.

[24] X. Li, A. Gray, D. Jiang, and X. Mao, "Sufficient and necessary conditions of stochastic permanence and extinction for stochastic logistic populations under regime switching," Journal of Mathematical Analysis and Applications, vol. 376, no. 1, pp. 1128, 2011.

[25] C. Zhu and G. Yin, "On hybrid competitive Lotka-Volterra ecosystems," Nonlinear Analysis. Theory, Methods \& Applications, vol. 71, no. 12, pp. el370-e1379, 2009.

[26] M. Liu and K. Wang, "Persistence and extinction of a stochastic single-specie model under regime switching in a polluted environment," Journal of Theoretical Biology, vol. 264, no. 3, pp. 934-944, 2010.

[27] M. Liu and K. Wang, "Asymptotic properties and simulations of a stochastic logistic model under regime switching," Mathematical and Computer Modelling, vol. 54, no. 9-10, pp. 2139-2154, 2011.

[28] Z. Wu, H. Huang, and L. Wang, "Stochastic delay population dynamics under regime switching-permanence and asymptotic estimation," Abstract and Applied Analysis. In press.

[29] X. Mao and C. Yuan, Stochastic Differential Equations with Markovian Switching, Imperial College Press, London, UK, 2006. 


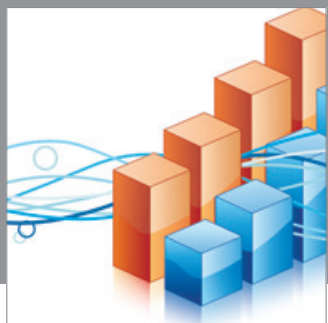

Advances in

Operations Research

mansans

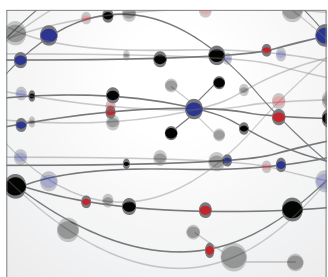

The Scientific World Journal
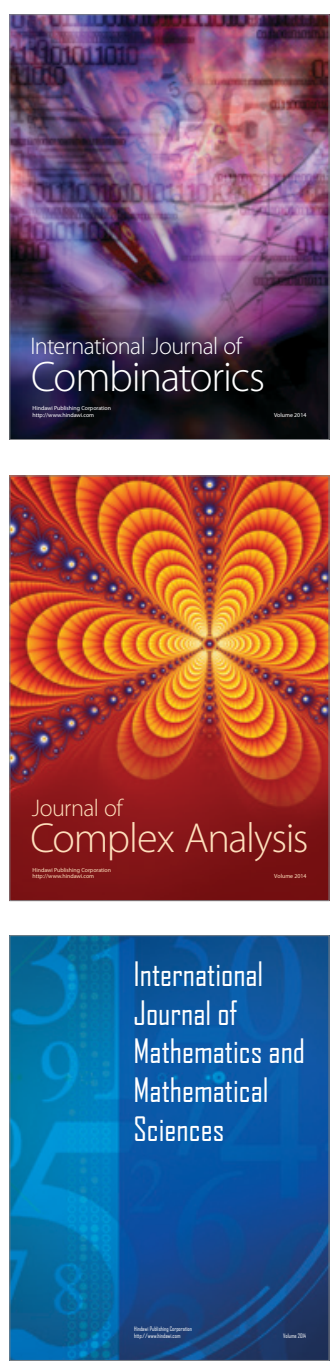
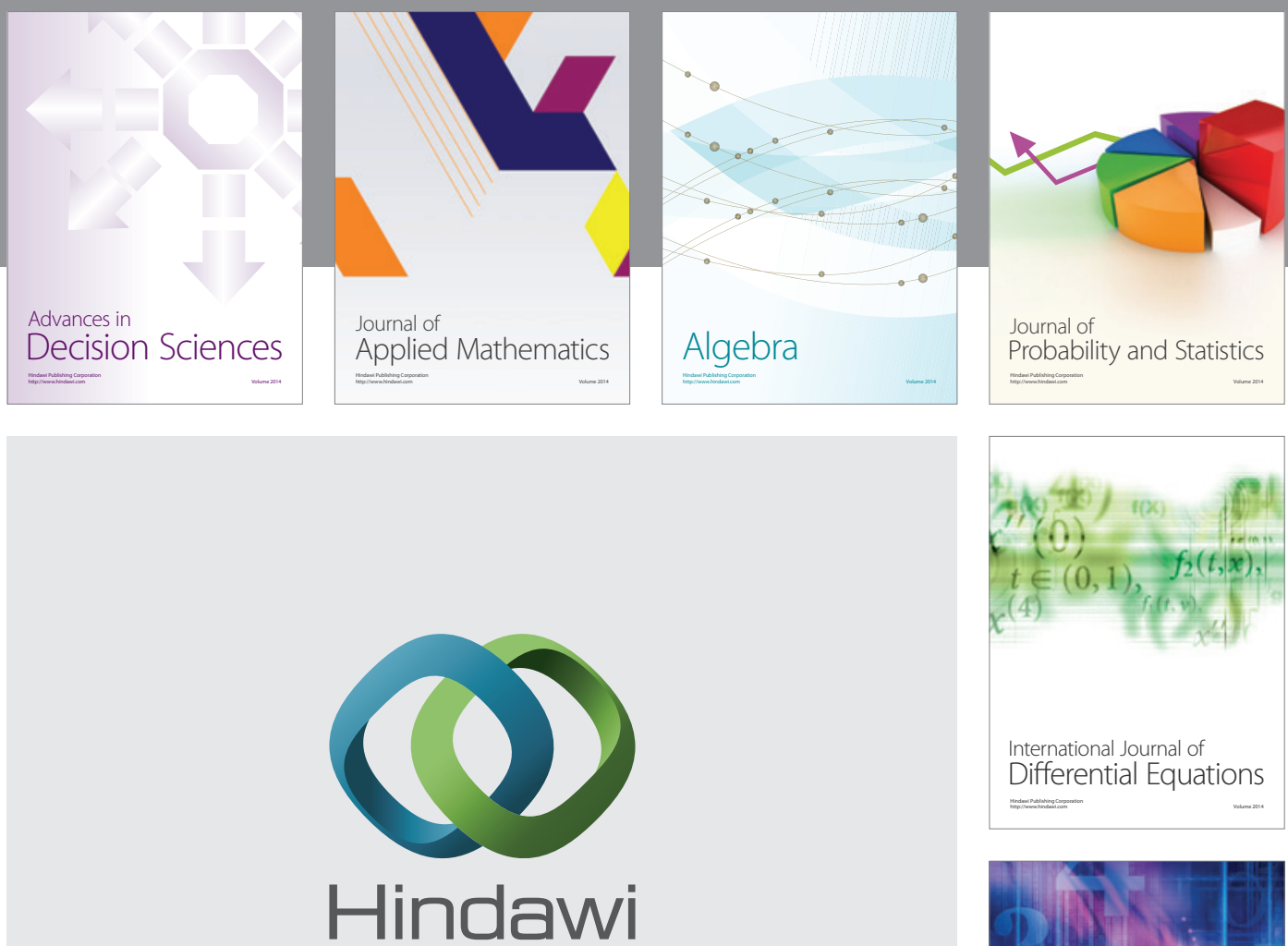

Submit your manuscripts at http://www.hindawi.com
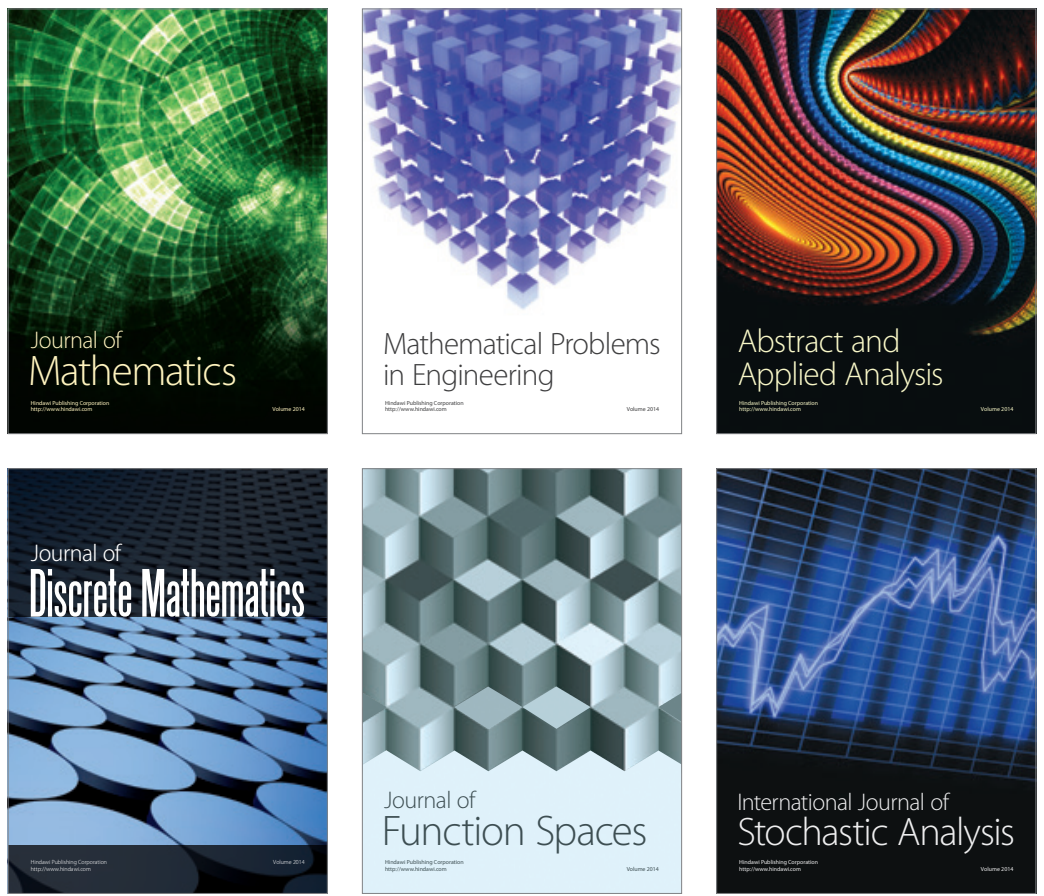

Journal of

Function Spaces

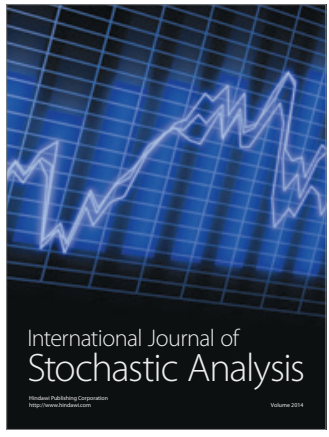

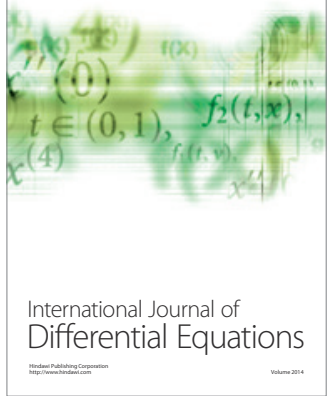
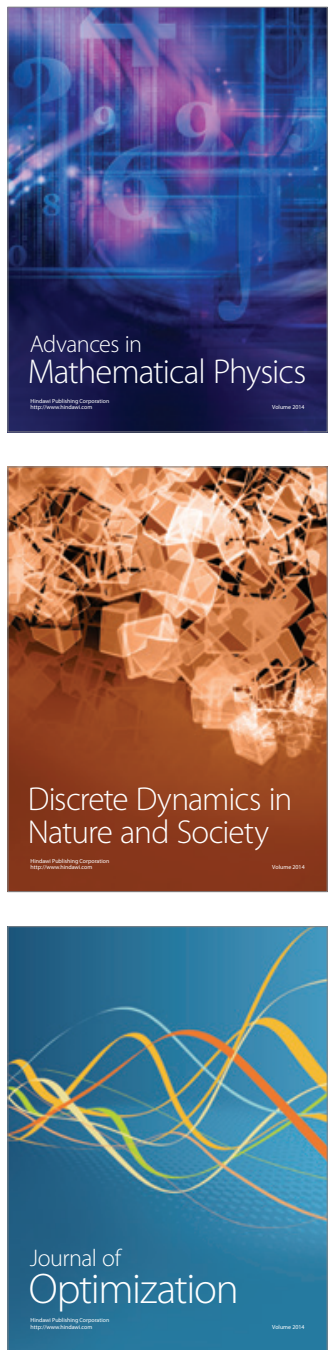